\title{
$\beta$ CaMKII Plays a Nonenzymatic Role in Hippocampal Synaptic Plasticity and Learning by Targeting $\alpha$ CaMKII to Synapses
}

\author{
Nils Z. Borgesius, Geeske M. van Woerden, Gabrielle H. S. Buitendijk, Nanda Keijzer, Dick Jaarsma, \\ Casper C. Hoogenraad, and Ype Elgersma \\ Department of Neuroscience, Erasmus University Medical Centre, 3015 GE, Rotterdam, the Netherlands
}

The calcium/calmodulin-dependent kinase type II (CaMKII) holoenzyme of the forebrain predominantly consists of heteromeric complexes of the $\alpha$ CaMKII and $\beta$ CaMKII isoforms. Yet, in contrast to $\alpha$ CaMKII, the role of $\beta$ CaMKII in hippocampal synaptic plasticity and learning has not been investigated. Here, we compare two targeted Camk $2 b$ mouse mutants to study the role of $\beta$ CaMKII in hippocampal function. Using a Camk $2 b^{-l-}$ mutant, in which $\beta$ CaMKII is absent, we show that both hippocampal-dependent learning and Schaffer collateral-CA1 long-term potentiation (LTP) are highly dependent upon the presence of $\beta$ CaMKII. We further show that $\beta$ CaMKII is required for proper targeting of $\alpha$ CaMKII to the synapse, indicating that $\beta$ CaMKII regulates the distribution of $\alpha$ CaMKII between the synaptic pool and the adjacent dendritic shaft. In contrast, localization of $\alpha$ CaMKII, hippocampal synaptic plasticity and learning were unaffected in the Camk $2 b^{A 303 R}$ mutant, in which the calcium/calmodulin-dependent activation of $\beta$ CaMKII is prevented, while the F-actin binding and bundling property is preserved. This indicates that the calcium/calmodulin-dependent kinase activity of $\beta$ CaMKII is fully dispensable for hippocampal learning, LTP, and targeting of $\alpha$ CaMKII, but implies a critical role for the F-actin binding and bundling properties of $\beta$ CaMKII in synaptic function. Together, our data provide compelling support for a model of CaMKII function in which $\alpha$ CaMKII and $\beta$ CaMKII act in concert, but with distinct functions, to regulate hippocampal synaptic plasticity and learning.

\section{Introduction}

Calcium/calmodulin-dependent kinase type II (CaMKII) is one of the most abundant proteins of the hippocampus, and its role in hippocampal plasticity and learning has been thoroughly investigated by pharmacological and genetic approaches. However, in the hippocampus there are two major isoforms of CaMKII, $\alpha$ and $\beta$, which cannot be distinguished using pharmacological approaches. In addition, almost all genetic approaches have focused on the $\alpha$ isoform (Silva et al., 1992; Mayford et al., 1996; Giese et al., 1998; Elgersma et al., 2002; Miller et al., 2002; Wang et al., 2008; Yamagata et al., 2009) (for an overview of CaMKII mutants, see Elgersma et al., 2004). Collectively, these studies have demonstrated that activation of $\alpha$ CaMKII is necessary for normal synaptic plasticity and learning, and, not surprisingly, its dereg-

Received Sept. 29, 2010; revised March 30, 2011; accepted April 8, 2011

Author contributions: N.Z.B., G.M.v.W., D.J., C.H., and Y.E. designed research; N.Z.B., G.M.v.W., G.H.B., N.K., D.J., and C.H. performed research; N.Z.B., G.M.v.W., D.J., and C.H. analyzed data; N.Z.B., G.M.v.W., and Y.E. wrote the paper.

This work was supported by grants from NWO-ZonMW and Neuro-Bsik to Y.E. We thank members of the Elgersma laboratory, Steven Kushner, and Roger Colbran for stimulating discussions and/or critically reading the manuscript. We thank Dmitri Andreev, Mehrnoush Agha Davoud Jolfaei, John Kongasan, Minetta Elgersma, and Hans van der Burg for technical support.

Correspondence should be addressed to Ype Elgersma, Department of Neuroscience, Erasmus University Medical Centre, Drive Molewaterplein 50, 3015 GE, Rotterdam, The Netherlands. E-mail: y.elgersma@erasmusmc.nl.

C. C. Hoogenraad's present address: Cell Biology, Faculty of Science, Utrecht University, Padualaan 8, $3584 \mathrm{CH}$ Utrecht, The Netherlands.

DOI:10.1523/JNEUROSCI.5105-10.2011

Copyright $\odot 2011$ the authors $\quad 0270-6474 / 11 / 3110141-08 \$ 15.00 / 0$ ulation can lead to severe cognitive impairments (van Woerden et al., 2007).

In the hippocampus, $\alpha$ CaMKII and $\beta$ CaMKII form a holoenzyme consisting of $\sim 12$ subunits in a 2:1 ratio (Brocke et al., 1999). $\alpha$ CaMKII and $\beta$ CaMKII are highly homologous, but they are encoded by two distinct genes (Camk2a and Camk2b, respectively) (Hudmon and Schulman, 2002a). The most noticeable difference between these isoforms is that $\beta$ CaMKII is able to bind to F-actin in an activity-controlled manner, through its extra domain in the variable region (Shen et al., 1998; Shen and Meyer, 1999). Two $\beta$ CaMKII subunits per holoenzyme are already sufficient to change the localization of the entire holoenzyme (Shen et al., 1998). Mainly due to these different actin-binding properties, $\alpha$ CaMKII and $\beta$ CaMKII were shown to have opposing effects on synaptic strength in cultured neurons (Thiagarajan et al., 2002). Interestingly, $\beta$ CaMKII not only binds to actin, but is also capable of bundling actin in a kinase-independent manner (O'Leary et al., 2006; Okamoto et al., 2007; Sanabria et al., 2009). This nonenzymatic bundling feature is likely achieved by single CaMKII oligomers binding to multiple actin filaments.

Studies addressing the role of $\beta$ CaMKII in synaptic plasticity and learning have only recently been initiated. Inducible overexpression of $\beta$ CaMKII in the dentate gyrus did not affect acquisition of hippocampal learning, but did affect the long-term consolidation of memories (Cho et al., 2007). Additionally, it was shown that the absence of $\beta$ CaMKII reverses the polarity of plasticity at cerebellar parallel fiber-Purkinje cell synapses and causes significant cerebellar learning deficits (van Woerden et al., 2009). 
The reversal of plasticity is caused in part by a nonenzymatic property of $\beta \mathrm{CaMKII}$, which prevents precocious activation of $\alpha$ CaMKII under low-calcium conditions.

Here we examined the role of $\beta$ CaMKII in hippocampal synaptic plasticity and learning using two different $\beta$ CaMKII mutants: (1) the Camk2 $b^{-1-}$ mouse, which does not express $\beta C a M K I I$; and (2) the Camk $2 b^{A 303 R}$ mouse, where a point mutation blocks calcium/calmodulin binding, selectively preventing its enzymatic activation, while preserving its ability to bind to actin (Shen and Meyer, 1999; Fink et al., 2003; Lin and Redmond, 2008). We found that the absence of $\beta$ CaMKII causes mislocalization of $\alpha$ CaMKII, impaired hippocampal synaptic plasticity, and impaired hippocampus-dependent learning. In contrast, these phenotypes were not present in the Camk2 $b^{A 303 R} \mathrm{mu}-$ tants, arguing that the actin binding and bundling function of $\beta$ CaMKII governs a major aspect of its synaptic function. These results strongly suggest an essential, but nonenzymatic role for $\beta$ CaMKII in hippocampal plasticity.

\section{Materials and Methods}

Generation of the Camk2b ${ }^{\mathrm{A} 303 \mathrm{R}}$ mutants. The Camk2 $b^{A 303 R}$ targeting construct was generated as follows. The Camk $2 b$ genomic sequence (ENSMUSG00000057897) was obtained from a public database (Ensembl) and used to design the primers for the targeting constructs. PCR fragments encompassing exon $6-11$ using $5^{\prime}$ primer: $5^{\prime}-$ GGTACCTGAGGAAGGTGCCAGCTCTGTCCC-3' and $3^{\prime}$ primer: $5^{\prime}-$ GTCGACCAGGGTAGTCACGGTTGTCC-3' ${ }^{\prime}(5.3 \mathrm{~kb}$; exon denotation according to ENSMUST00000019133) and exon 11-12 using $5^{\prime}$ primer: $5^{\prime}$-GCGGCCGCCTGTTAAAGGAATGGTTCTC-3' and $3^{\prime}$ primer: $5^{\prime}-$ ATGCATCTAAAAGGCAGGCAGGATGATCTGC-3' (6 kb) were amplified using High Fidelity Taq Polymerase (Roche) on ES cell genomic DNA and cloned on either site of a PGK (phosphoglycerate kinase)-Neomycin selection cassette. All exons were sequenced to verify that no mutations were introduced accidentally. Site-directed mutagenesis was used to introduce the point mutation Ala303Arg. For counter selection, a gene encoding diphtheria toxin chain A (DTA) was inserted at the $5^{\prime}$ of the targeting construct. The targeting construct was linearized and electroporated into embryonic day 14 (E14) ES cells (derived from 129P2 mice). Cells were cultured in BRL cellconditioned medium in the presence of leukemia inhibitory factor. After selection with G418 $(200 \mu \mathrm{g} / \mathrm{ml})$, targeted clones were identified by PCR (long-range PCR from neomycin resistance gene to the region flanking the targeted sequence). A clone with normal karyotype was injected into blastocysts of C57BL/6 mice. Male chimeras were crossed with female C57BL/6 mice (Harlan). The resulting F1 heterozygous mice (in the 129P2-C57BL/6 background) were used to generate F2 homozygous mutants and wild-type littermate controls. These mice, of either sex, were used for all the behavioral and electrophysiological experiments. The experimenter was blind for the genotype, but homozygous mice were easily recognizable by the ataxic gait. Therefore, a second person blind to the genotype also analyzed the data. Mice were housed on a $12 \mathrm{~h}$ light/dark cycle with food and water available ad libitum, and were used between 2 and 6 months of age for all experiments described (including electrophysiology). All animal procedures were approved by a Dutch Ethical Committee for animal experiments.

Western blot. Lysates were prepared by quick dissection of the brain and by homogenization of the brain tissue in lysis buffer (10 mM TRIS$\mathrm{HCl} 6.8$, 2.5\% SDS, 2 mM EDTA, and protease and phosphatase inhibitor cocktails; Sigma). The concentration of the lysates was adjusted to 1 $\mathrm{mg} / \mathrm{ml}$. Ten micrograms was used for Western blot analysis. Western blots were probed with antibodies directed against $\alpha$ CaMKII (MAB3119, 1:10,000; Millipore Bioscience Research Reagents), $\beta$ CaMKII (CB- $\beta 1$, 1:10,000; Zymed), Ph-T286/T87 CaMKII antibody (1:5000; catalog \#06881, Millipore Cell Signaling Solutions), and actin (MAB1501R, 1:2000; Millipore Bioscience Research Reagents). Blots were stained using Enhanced ChemoLuminescence (catalog \#32106, Pierce). Western blot quantification was performed using NIH-Image.

Immunocytochemistry. Immunocytochemistry was performed on freefloating $40-\mu \mathrm{m}$-thick frozen sections using a standard avidin-biotin- immunoperoxidase complex method (ABC, Vector Laboratories) with $\beta$ CaMKII (CB-b1, 1:2000; Zymed) as the primary antibody and diaminobenzidine $(0.05 \%)$ as the chromogen (Hansel, 2006). For gross brain morphology, sections were stained with thionin.

Dendritic arborization. Golgi-Cox staining on unfixed hippocampi of three Camk $2 b^{-1-}$ mutants and three wild-type mice was performed using the FD Rapid GolgiStain Kit (FD NeuroTechnologies), according to the manufacturers' instructions. Sagittal sections, $100 \mu \mathrm{m}$ thick, were cut on a microtome with cryostat adaptations. Pyramidal cell counting and selection for further detailed analysis was done by two independent observers who were both blind for genotype. A calibration grid was used to count the number of spines per $10 \mu \mathrm{m}$, using a $40 \times$ objective.

Primary hippocampal cultures and immunohistochemistry. $\beta$ CaMKII or $\beta$ CaMKII-A303R heterozygous mice were crossed, and wild-type, heterozygous, and knock-out hippocampal neuron cultures were prepared from brains of single E18 embryos out of mixed genotype litters. Mouse hippocampal neurons were isolated and prepared as described previously (Goslin and Banker, 1991). In short, the two hippocampi were removed from the embryonic brain, collected in $1 \mathrm{ml}$ of DMEM on ice, washed two times with $1 \mathrm{ml}$ of DMEM, and incubated in trypsin/EDTA solution (Invitrogen ) at $37^{\circ} \mathrm{C}$ for $15 \mathrm{~min}$. After washing with $1 \mathrm{ml}$ of DMEM, the cells were resuspended in neurobasal medium (NB) supplemented with $2 \%$ B27, $1 \%$ penicillin/streptomycin, and $1 \%$ glutamax (Invitrogen ), and dissociated using a gently flamed Pasteur pipette. Neurons were plated in a small drop on poly-L-lysine- $(100 \mu \mathrm{g} / \mathrm{ml}$, Sigma) and laminin-coated (50 $\mu \mathrm{g} / \mathrm{ml}$, Sigma) $15 \mathrm{~mm}$ glass coverslips at a density of 75,000 per coverslip in 12 well plates. After $2 \mathrm{~h}, 1 \mathrm{ml}$ of NB supplemented with $2 \% \mathrm{~B} 27,1 \%$ penicillin/streptomycin, and $1 \%$ glutamax was added to the coverslips. Neuronal activity was suppressed by treating the neuronal cultures with TTX for $24 \mathrm{~h}$ ( $2 \mu \mathrm{M}$, Sigma).

For immunohistochemistry, two [guanylate kinase domain-associated protein (GKAP)] or three (bassoon) independent cultures of $14 \mathrm{~d}$ in vitro (DIV14) neurons were fixed for $10 \mathrm{~min}$ with $4 \%$ formaldehyde/4\% sucrose in PBS at room temperature (Jaworski et al., 2009). After fixation, the cells were washed two times in PBS for $30 \mathrm{~min}$ at room temperature, and were incubated with primary antibodies in GDB buffer $(0.2 \%$ BSA, $0.8 \mathrm{M} \mathrm{NaCl}$, $0.5 \%$ Triton X-100, $30 \mathrm{~mm}$ phosphate buffer, $\mathrm{pH} 7.4$ ) overnight at $4^{\circ} \mathrm{C}$. The following primary antibodies were used: rabbit anti-bassoon (Synaptic Systems), rabbit anti-GKAP (Santa Cruz Biotechnology), and mouse antiaCaMKII (Sigma). Phalloidin (Invitrogen) was used to stain filamentous actin (F-actin). Neurons were then washed three times in PBS for $30 \mathrm{~min}$ at room temperature and incubated with Alexa488- and Alexa568-conjugated secondary antibodies (Invitrogen) in GDB for $2 \mathrm{~h}$ at room temperature, and washed three times in PBS for 30 min. Slides were mounted using Vectashield mounting medium (Vector Laboratories). Confocal images were acquired using a LSM510 confocal microscope (Zeiss) with a $40 \times$ or $63 \times$ oil objective.

For quantification, confocal images of four to five neurons obtained from two to three independent experiments were obtained, with sequential acquisition settings at the maximal resolution of the microscope $(1024 \times 1024$ pixels). Each image was a $z$-series of six to eight images each averaged two times was chosen to cover the entire region of interest from top to bottom. The resulting $z$-stack was "flattened" into a single image using maximum projection. Images were not further processed and were of similar high quality to the original single planes. The confocal settings were kept the same for all scans when fluorescence intensity was compared. Morphometric analysis, quantification, and colocalization were performed using MetaMorph software (Universal Imaging Corporation). The ratio of $\alpha$ CaMKII in the synapse over $\alpha$ CaMKII in the shaft was calculated by measuring the average intensity of the fluorescent $\alpha$ CaMKII signal in the synapses and the shaft.

Fear conditioning. Fear conditioning was performed in a conditioning chamber (Medical Associates) equipped with a grid floor via which the footshock could be administered. Each mouse was placed inside the conditioning chamber for $180 \mathrm{~s}$. A footshock $(2 \mathrm{~s}, 0.4 \mathrm{~mA})$ was delivered $148 \mathrm{~s}$ after placement in the chamber. Twenty-four hours later, context-dependent freezing was measured during 3 min.

Electrophysiology. After the animals had been killed, sagittal slices (400 $\mu \mathrm{m})$ were obtained and submerged in ice-cold artificial CSF (ACSF) 


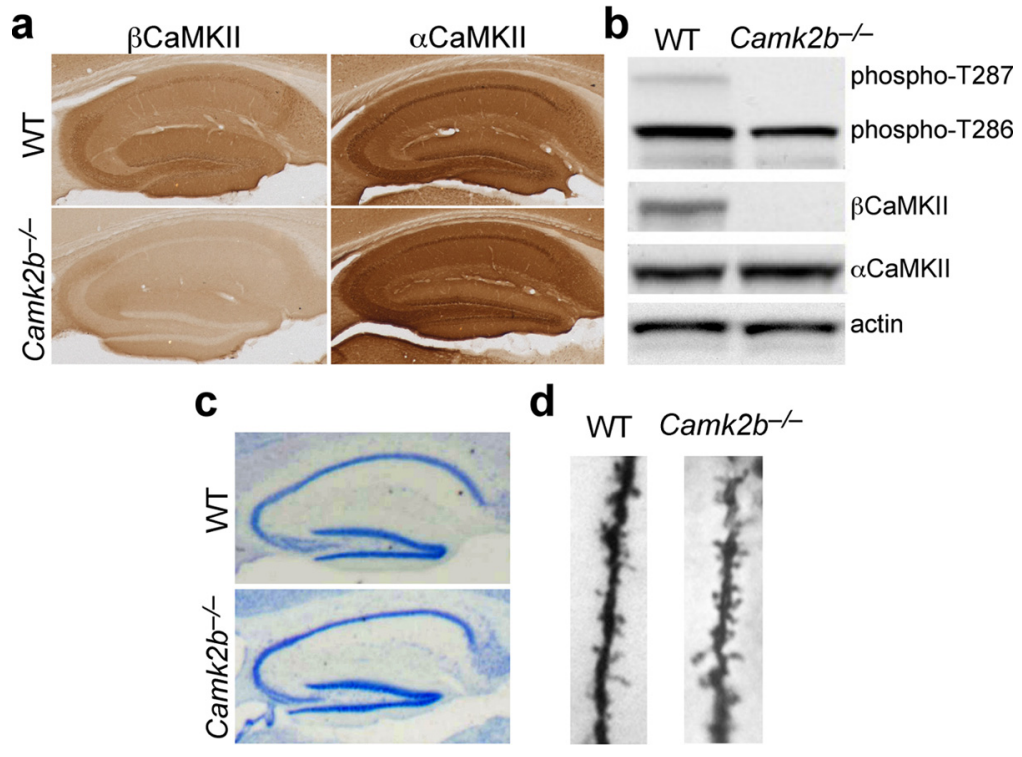

Figure 1. Morphological and molecular analysis of the Camk2 $b^{-1-}$ mice. $\boldsymbol{a}$, Immunocytochemistry analysis using $\alpha$ CaMKII- and $\beta$ CaMKII-specific antibodies shows complete absence of $\beta$ CaMKII in the hippocampus, with no apparent change in $\alpha$ CaMKII expression. $\boldsymbol{b}$, Western blot analysis using $\alpha$ CaMKII- and $\beta$ CaMKII-specific antibodies reveals no change in the levels of $\alpha$ CaMKII protein or in the levels of $\alpha$ CaMKII-T286 phosphorylation. In contrast, $\beta$ CaMKII protein and $\beta$ CaMKII-T287 phosphorylation are completely absent. $c$, Thionin staining shows no apparent morphological change in the hippocampus of Camk $2 b^{-1-}$ mice compared with wild-type mice. $\boldsymbol{d}$, Quantification of Golgi analysis of the hippocampal pyramidal cells does not reveal any difference in spine density.

using a vibratome, and hippocampi were dissected out. These sagittal hippocampal slices were maintained at room temperature for at least $1.5 \mathrm{~h}$ to recover before experiments were initiated. Then they were placed in a submerged recording chamber and perfused continuously at a rate of $2 \mathrm{ml} / \mathrm{min}$ with ACSF equilibrated with $95 \% \mathrm{O}_{2}, 5 \% \mathrm{CO}_{2}$ at $31^{\circ} \mathrm{C}$. ACSF contained the following (in $\mathrm{mm}$ ): $120 \mathrm{NaCl}, 3.5 \mathrm{KCl}, 2.5 \mathrm{CaCl}_{2}, 1.3$ $\mathrm{MgSO}_{4}, 1.25 \mathrm{NaH}_{2} \mathrm{PO}_{4}, 26 \mathrm{NaHCO}_{3}$, and 10 D-glucose. Extracellular recordings of field EPSPs (fEPSPs) were made in CA1 stratum radiatum with platinum (Pt)/iridium (Ir) electrodes (Frederick Haer Company). A bipolar Pt/Ir was used to stimulate Schaffer collateral/commissural afferents with a stimulus duration of $100 \mu$ s. Stimulus-response curves were obtained at the beginning of each experiment, $20 \mathrm{~min}$ after placing the electrodes. Long-term potentiation (LTP) was evoked using the following two different tetani: (1) $100 \mathrm{~Hz}$ ( 1 train of $1 \mathrm{~s}$ at $100 \mathrm{~Hz}$ ); and (2) 200 $\mathrm{Hz}$ ( 4 trains of $0.5 \mathrm{~s}$, spaced by $5 \mathrm{~s}$ ). Both protocols were performed at one-third of the maximum fEPSP. fEPSP measurements were done once per minute. Potentiation was measured as the normalized increase of the mean fEPSP slope for the duration of the baseline. Only stable recordings were included, and this judgment was made blind to genotype. Average LTP was defined as the mean last 10 min of the normalized fEPSP slope.

Statistical analysis. All data are presented as means \pm SEM and were tested for normality of distribution using the D'Agostino-Pearson test. If normality of distribution was violated or sample size was too small to determine normality, we used the Mann-Whitney $U$ test. In all other cases, an appropriate $t$ test was used to analyze differences between genotypes for spine density, freezing time, and LTP induction (based on the average of the last $10 \mathrm{~min}$ ). A repeated-measures ANOVA was used to analyze differences between genotypes for fiber volley and fEPSP slope, even when in some cases the distribution was not normal because there is no nonparametric alternative for the repeated-measures ANOVA.

\section{Results}

Camk $2 b^{-/-}$mice show normal hippocampal morphology and no change in $\alpha$ CaMKII protein levels and autophosphorylation

Generation of the Camk2b $b^{-1-}$ mouse has been described previously (van Woerden et al., 2009). Using immunohistochemistry and Western blot, we confirmed the absence of $\beta$ CaMKII in the hippocampus of the Camk2b $b^{-1-}$ mouse (Fig. 1a,b). Since in vitro experiments showed that upregulation of $\beta$ CaMKII causes downregulation of $\alpha$ CaMKII (Thiagarajan et al., 2002), we tested whether the absence of $\beta$ CaMKII caused upregulation of $\alpha$ CaMKII in vivo. However, we did not observe a change in $\alpha$ CaMKII protein levels (wild-type mice: $100 \pm 5.4, n=6$; Camk $2 b^{-1-}$ mice: $91.5 \pm 6.3, n=7$; Mann-Whitney $U$ test, $U=13.00, p=0.29$ ) (Fig. 1b), nor was there a significant change in basal levels of $\alpha$ CaMKII Thr ${ }^{286}$ phosphorylation (wild-type mice:100 \pm $17.1 n=6$; Camk $2 b^{-1-}$ mice: $92.7 \pm 13.9$ $n=7$; Mann-Whitney $U$ test, $U=20.00$, $p=0.95)$ (Fig. 1b). These data show that in $C a m k 2 b^{-l-}$ mice $\beta$ CaMKII is absent and that protein expression and basal levels of autophosphorylation of $\alpha$ CaMKII are unaltered.

Previous studies showed that upregulation or downregulation of $\beta$ CaMKII in vitro caused respectively an increased or decreased dendritic arborization, suggesting that $\beta$ CaMKII might be critical for normal dendritic development in vivo (Fink et al., 2003). Therefore, we performed a detailed examination of the hippocampus using thionin staining. However, we found no evidence of significant changes in hippocampal structure at the light microscopy level (Fig. 1c). Furthermore, we investigated the morphology of the CA1 pyramidal cells using Golgi-Cox staining. We found no significant change in the density of spines (wild-type mice: $6.35 \pm 0.36 n=$ 15 cells from 3 mice; Camk $2 b^{-1-}$ mice: $7.07 \pm 0.61 n=15$ from 3 mice; unpaired two-tailed $t$ test, $t_{(28)}=1.8, p=0.09$ ) (Fig. $1 d$ ), which is consistent with our previous findings for the cerebellum (van Woerden et al., 2009). Together, these data show that gross neuronal development is preserved in Camk $2 b^{-1-}$ mice.

\section{$\alpha$ CaMKII is mislocalized in Camk2b $b^{-/-}$neurons}

Since $\beta$ CaMKII is able to bind F-actin in an activity-controlled manner (Shen et al., 1998; Shen and Meyer, 1999) and pharmacologically induced changes in actin bundling have a large effect on CaMKII delivery in spines (Allison et al., 2000; Okamoto et al., 2004 ), it is possible that $\beta$ CaMKII can change actin dynamics and the localization of the CaMKII holoenzyme. Therefore, we hypothesized that absence of $\beta$ CaMKII might result in abnormalities in synaptic localization of $\alpha$ CaMKII. However, using brain sections for quantitative analysis of changes in localization of $\alpha$ CaMKII is challenging, because of its ubiquitous distribution, and its ability to self-aggregate and redistribute depending on multiple factors including fixation conditions (Tao-Cheng et al., 2002). Hence, we investigated the localization of endogenous $\alpha$ CaMKII by immunostaining of neuronal cultures obtained from E18 hippocampal neurons from Camk $2 b^{-1-}$ and wild-type mice. Neurons were fixed at DIV14 and stained with an antibody against bassoon (a marker for the presynaptic active zone), GKAP (a marker for the postsynaptic density), phalloidin (which stains F-actin), and an antibody against $\alpha$ CaMKII (Fig. 2). Whereas $\alpha$ CaMKII showed a striking synaptic labeling in wild-type neurons, this was clearly less pronounced in the Camk $2 b^{-1-}$-derived neurons. To quantify this, we measured the ratio of $\alpha \mathrm{CaMKII}$ in 
the synapse (colocalizing with bassoon or GKAP) to $\alpha$ CaMKII in the dendritic shaft, and found that this ratio was reduced by $>40 \%$ in the Camk2 $b^{-1-}$ neurons when measured over bassoon ( $\alpha$ CaMKII $_{\text {synapse }}$ ' $\alpha \mathrm{CaMKII}_{\text {shaft }}$ ratio: wild-type neurons, $3.3 \pm 0.2, n=15$; Camk2 $b^{-1-}$ neurons, $2.1 \pm 0.2, n=14$; Mann-Whitney $U$ test, $U=17.00, p=0.0002$ ) (Fig. $2 a$ ), and by $60 \%$ when measured over GKAP ( $\alpha \mathrm{CaMKII}_{\text {spine }} / \alpha \mathrm{CaMKII}_{\text {shaft }}$ ratio: wildtype neurons, $3.42 \pm 0.16, n=10$; Camk2 $b^{-1-}$ neurons, $1.50 \pm 0.14 n=10$; Mann-Whitney $U$ test, $U=8.00, p=$ 0.001) (Fig. 2b). This distinct difference was still observed after decreasing or increasing neuronal activity of Camk $2 b^{-1-}$ neuronal cultures with tetrodotoxin or bicuculline for $24 \mathrm{~h}$, as neither treatment changed the $\alpha$ CaMKII localization significantly ( $\alpha \mathrm{CaMKII}_{\text {synapse }} / \alpha \mathrm{CaMKII}$ shaft $\mathrm{ra}$ tio: control medium, $1.66 \pm 0.19, n=5$; tetrodotoxin, $1.74 \pm 0.18, n=5$; bicuculline, $1.80 \pm 0.13, n=6$; ANOVA $F_{(2,13)}=$ $0.05 ; p=0.96)$. This indicates that the changes were not due to differences in spontaneous activity between the cultures. In addition, even though the cultures were derived from E18 neurons, the ratio of $\alpha \mathrm{CaMKII} / \beta \mathrm{CaMKII}$ in wild-type DIV14 cultures was comparable to the ratio observed in the adult mouse hippocampus (Fig. 2d). Together, these findings highlight the importance of $\beta$ CaMKII in regulating the distribution of endogenous $\alpha$ CaMKII between the synaptic pool and the adjacent dendritic shaft.

\section{Camk $2 b^{-/-}$mice show impaired LTP}

Given that overall neuronal morphology was unaffected in Camk2 $b^{-1-}$ mutants with an impaired localization of $\alpha$ CaMKII into dendritic spines, we examined the functional implications by investigating synaptic plasticity at the hippocampal CA1 synapse. Using extracellular recordings in acute hippocampal slices, we focused on the Schaffer-collateral pathway given the large literature implicating this synapse in many forms of hippocampus-dependent learning and memory. No significant impairment in basal synaptic transmission was observed in the Camk2 $b^{-1-}$ mice (Fig. 3a), with significant changes in neither fiber volley (repeated-measures ANOVA, $F_{(1,135)}=0.22 ; p=0.64$ ) nor fEPSP slope (repeatedmeasures ANOVA, $\left.F_{(1,135)}=1.4 ; p=0.23\right)$. However, we found a significant deficit in LTP in Camk $2 b^{-1-}$ mutants compared with wild-type littermates (wild-type mice, 152.6 $\pm 7.7, n=27$; Camk $2 b^{-1-}$ mice, $119.3 \pm 4.4, n=21$; Mann-Whitney $U$ test, $U=130.00, p=0.0015$ ) (Fig. $3 b$ ), indicating that $\beta$ CaMKII plays an essential role in synaptic plasticity at the hippocampal Schaffer-collateral pathway. Notably, the LTP deficit in the Camk $2 b^{-1-}$ mice induced by a $100 \mathrm{~Hz} / 1 \mathrm{~s}$ tetanus, is as severe as
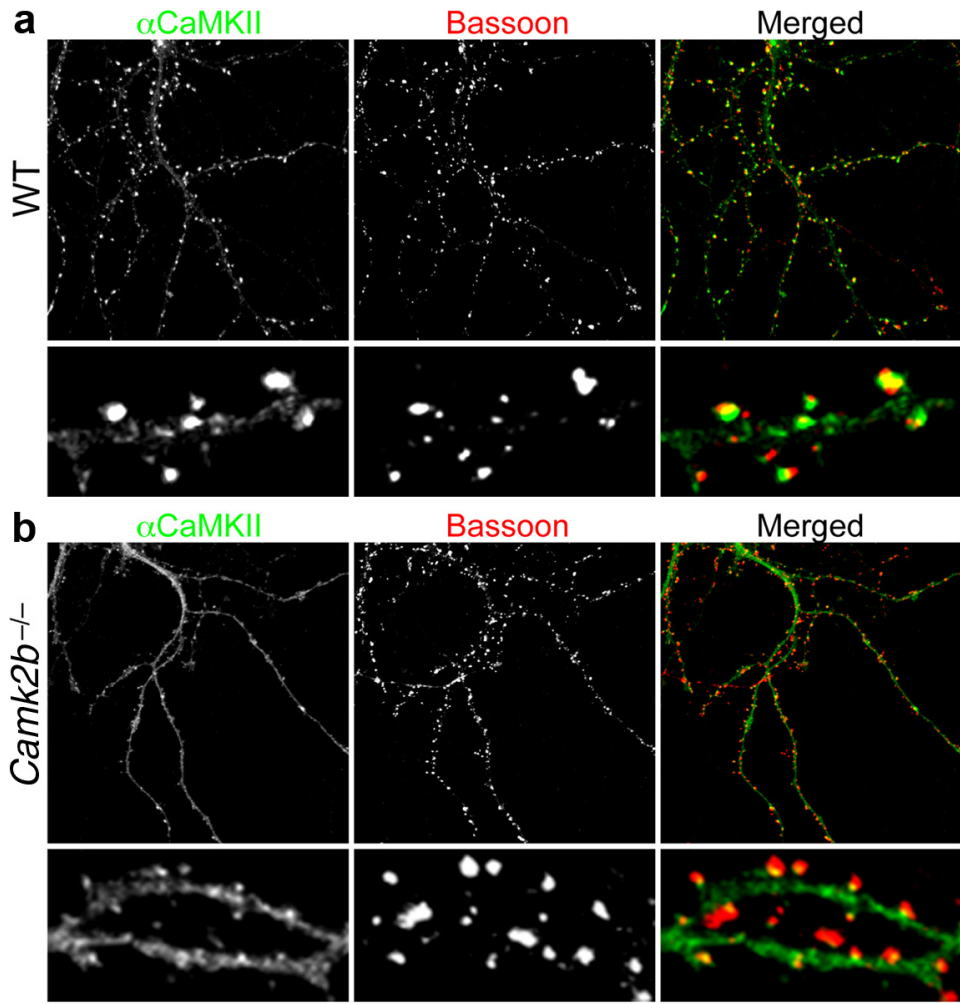

Merged
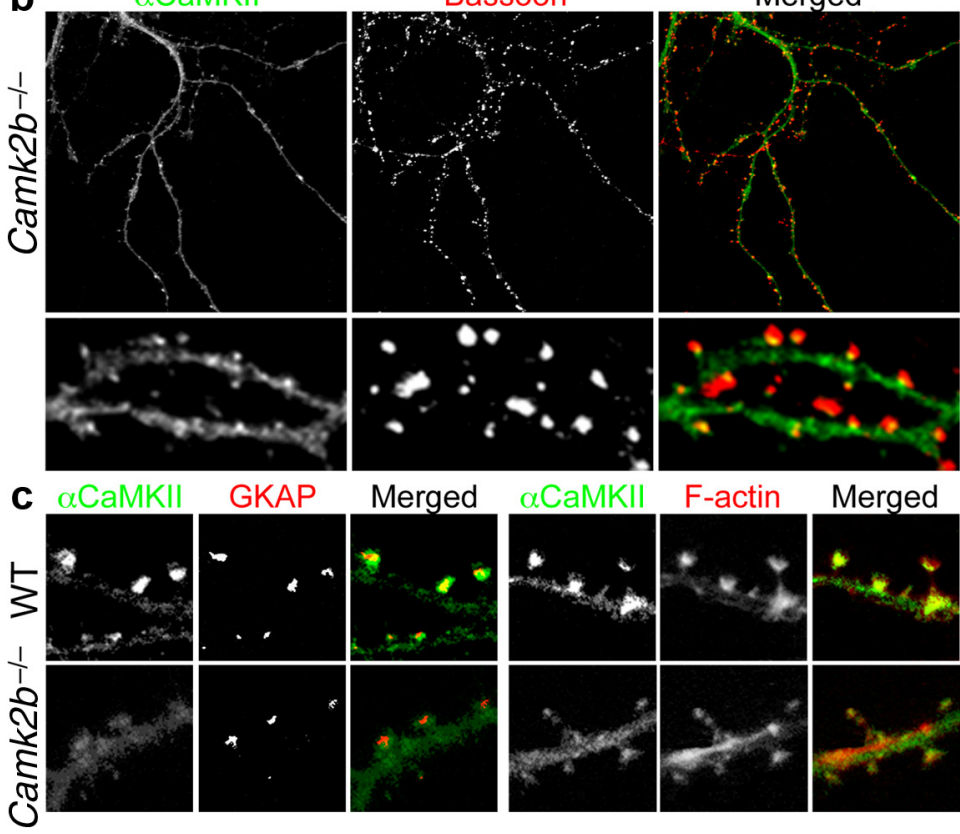

Merged

Figure 2. Impaired $\alpha$ CaMKII targeting to dendritic spines in Camk $2 b^{-1-}$ mice. $\boldsymbol{a}$, Representative images of cultured wild-type hippocampal neurons (top), and an enlargement of a dendritic segment (bottom), labeled with $\alpha$ (aMKII (green) and bassoon (red), and their colocalization (merged). $\boldsymbol{b}$, Representative images of cultured Camk2 $b^{-1-}$ hippocampal neurons (top), and an showing reduced targeting of $\alpha$ CaMKII to synapses in Camk2 $b^{-1-}$ hippocampal neurons. c, Representative images of wild-type (top row) and Camk2b $b^{-1-}$ (bottom row) hippocampal dendritic segments, labeled with $\alpha$ CaMKII (green) and GKAP (red), and their colocalshowing reduced targeting of $\alpha$ CaMKII to spines in Camk $2 b^{-1-}$ hippocampal neurons. $\boldsymbol{d}$, Western blot shows comparable ratio of $\alpha$ CaMKII $(\alpha)$ to $\beta$ CaMKII $(\beta)$ in E18-derived wild-type cultured neurons on DIV14 compared with a lysate from an adult wild-type mouse.

after the loss of the far more abundant $\alpha$ CaMKII (Elgersma et al., 2002). Moreover, it should be noted that hippocampal LTP is unaffected in the heterozygous Camk2a mutant (Frankland et al., 2001; Elgersma et al., 2002), which shows a larger decrease in total CaMKII level compared with the homozygous Camk2b mutant. This indicates that the LTP deficit of the Camk2 $b^{-1-}$ mice cannot only be explained in terms of loss of CaMKII activity.

It has previously been shown that changes in the F-actin/Gactin equilibrium affect $\alpha$ CaMKII localization (Allison et al., 2000; Okamoto et al., 2004) and that stimulation of NMDA receptors affects CaMKII localization as well as the F-actin/G-actin equilibrium (Shen and Meyer, 1999; Okamoto et al., 2004). Together, these findings support a model in which CA1 LTP is 

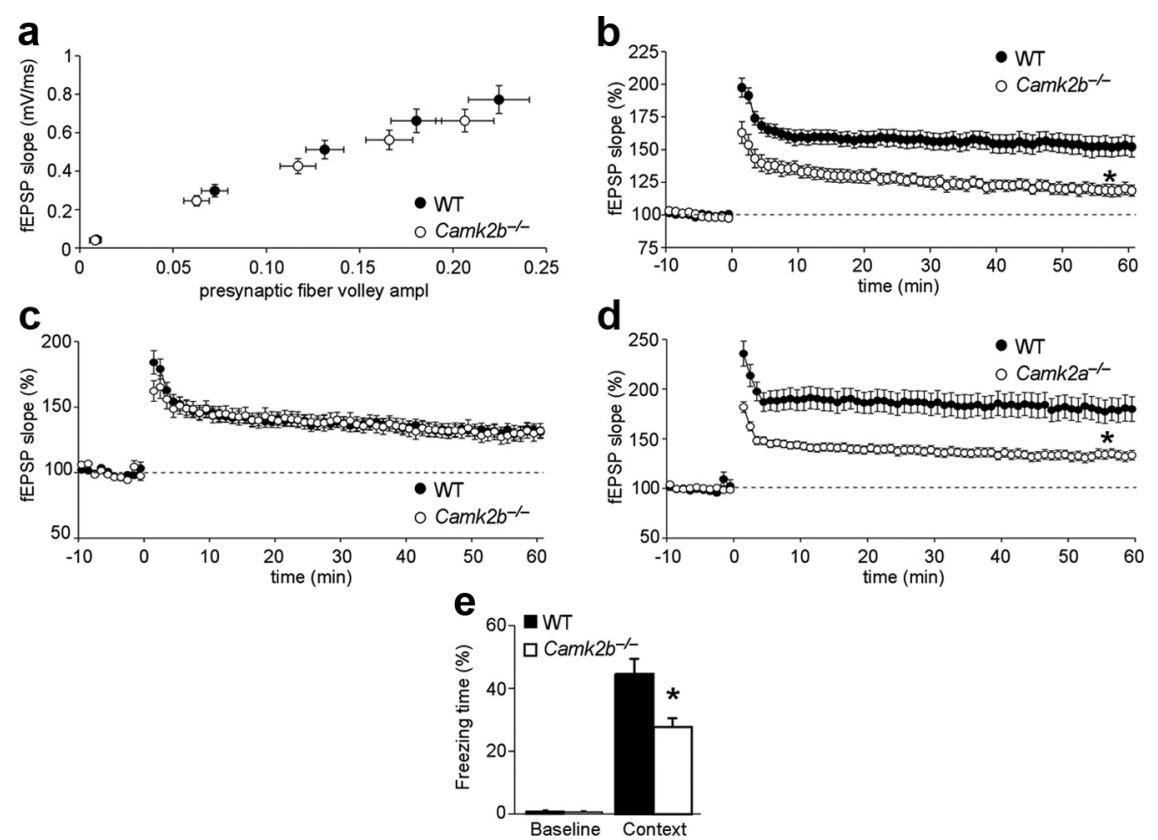

Figure 3. Hippocampal plasticity and learning in Camk $2 b^{-1-}$ mice. $a$, Camk $2 b^{-1-}$ mice show normal synaptic transmission (wild-type, $n=74$; Camk2 $b^{-1-}, n=63$ ). $\boldsymbol{b}$, Camk2 $b^{-1-}$ mice show impaired $100 \mathrm{~Hz}$ LTP (wild-type, $n=27$; Camk2 $b^{-1-}$, $n=21) . \boldsymbol{c}$, Camk $2 b^{-1-}$ mice show normal $200 \mathrm{~Hz}$ LTP (wild-type, $\left.n=14 ; \mathrm{Camk}_{2} b^{-1-}, n=12\right)$. $\boldsymbol{d}$, The $200 \mathrm{~Hz}$ LTP is dependent on $\alpha$ CaMKII, as Camk2a $a^{-1-}$ mice show impaired $200 \mathrm{~Hz}$ LTP (wild-type, $n=12$; Camk2a $a^{-1-}, n=18$ ).e, Impaired contextual fear conditioning in Camk $2 b^{-1-}$ mice. Percentage of time spent freezing during training before the footshock (Pre) and $24 \mathrm{~h}$ after conditioning (Post) indicates reduced postshock freezing, while preshock freezing is normal (wild-type, $n=10$; Camk2 $b^{-1-}$, $n=10)$. Error bars represent SEM; asterisks indicate a significant difference between mutant and control.

highly dependent upon proper targeting of $\alpha$ CaMKII into spines, for which $\beta$ CaMKII is required. If the LTP deficit is due to mislocalization of $\alpha$ CaMKII, it is conceivable that the LTP deficit can be rescued by using a stronger LTP-inducing protocol (4 trains of $200 \mathrm{~Hz}$ for $0.5 \mathrm{~s}$, spaced $5 \mathrm{~s}$ apart) (Grover and Teyler, 1990). Indeed, using this stimulation protocol, LTP is normal in Camk $2 b^{-1-}$ mice, confirming the functional implications of the altered CaMKII localization (wild-type mice, 131.6 $\pm 4.4, n=14$; Camk $2 b^{-1-}$ mice, $129.7 \pm 5.7, n=12$; unpaired two-tailed $t$ test, $t_{(24)}=0.36, p=0.72$ ) (Fig. 3c). To confirm that this $200 \mathrm{~Hz}$ LTP protocol requires $\alpha \mathrm{CaMKII}$, we also performed this experiment in Camk2a $a^{-1-}$ mice. Indeed, Camk2a $a^{-1-}$ mice show a significant impairment (wild-type mice, $179.6 \pm 13.3, n=12$; Camk $2 a^{-1-}$, $133.3 \pm 4.8, n=18$; Mann-Whitney $U$ test, $U=36.00, p=$ 0.0025) (Fig. 3d), indicating that this LTP protocol is indeed dependent on CaMKII activity. Together, these results show that the LTP deficit in the Camk $2 b^{-1-}$ mice can be overcome by a strong LTP-inducing protocol. These findings are consistent with, but not a proof of, the hypothesis that the deficit in hippocampal synaptic plasticity is a result of a failure to properly target $\alpha$ CaMKII into dendritic spines of the Camk $2 b^{-1-}$ mouse.

\section{Camk $2 b^{-/-}$mice show impaired hippocampus-dependent learning}

To test whether the mislocalization of CaMKII in the Camk2 $b^{-1-}$ mouse also affected hippocampal learning, we made use of contextual fear conditioning. In this task, mice are conditioned to associate a certain context with a mild, aversive footshock. Learning is assessed by measuring freezing behavior (i.e., the cessation of all movement except respiration), which is a natural expression of fear in mice. Camk $2 b^{-1-}$ mutants did not differ from their wild-type littermates in preshock baseline freezing behavior (wild-type mice, $0.9 \pm 0.2 \%, n=11$; Camk $2 b^{-1-}$ mice, $0.7 \pm 0.2 \%, n=9$; twotailed $t$ test, $t_{(18)}=0.72, p=0.48$ ) (Fig. $3 e)$, but showed significantly less freezing in the $24 \mathrm{~h}$ long-term memory test, demonstrating an impairment of hippocampusdependent memory (wild-type mice, $44.8 \pm 4.6 \%, n=11$; Camk $2 b^{-1-}$ mice, $27.8 \pm 2.7 \%, n=9$; two-tailed $t$ test, $t_{(18)}=$ 3.0, $p=0.0079$ ) (Fig. 3e).

\section{Generation and characterization of the Camk $2 \boldsymbol{b}^{A 303 R}$ mouse}

The results above suggest that $\beta$ CaMKII strongly influences hippocampal plasticity by regulating $\alpha$ CaMKII localization into dendritic spines. However, the affinity of $\beta$ CaMKII for calcium/calmodulin is nearly 10 -fold higher than $\alpha$ CaMKII, and the sensitivity range of the heteromeric holoenzyme is dependent on the ratio of $\alpha$ to $\beta$ subunits (De Koninck and Schulman, 1998; Brocke et al., 1999). Hence, we cannot rule out that the deficits in CA1 LTP and hippocampus-dependent learning are caused by the loss of the enzymatic activity of $\beta$ CaMKII, rather than the abnormal localization of $\alpha$ CaMKII. Therefore, we sought to distinguish between these possibilities using a well described mutation of $\beta$ CaMKII (A303R), which prevents kinase activation (by interfering with calcium/calmodulin binding) while preserving F-actin binding and bundling (Shen and Meyer, 1999; Fink et al., 2003; O'Leary et al., 2006). In addition, the $\beta$ CaMKII-A303R protein does not show a dominant-negative effect on dendritic arborization in vitro, in contrast to cells expressing the catalytically dead $\beta$ CaMKII-K42R protein, which cannot bind ATP (Fink et al., 2003). Accordingly, this mutation elegantly permits us to dissect the requirement of $\beta$ CaMKII kinase activity and F-actin bundling on LTP and learning.

We created a knock-in mutant of the Camk2b gene, substituting alanine ${ }^{303}$ for arginine (A303R) (Fig. 4a,b). Immunostaining and Western blot analysis of brains of homozygous point mutants (designated as Camk2 $b^{A 303 R}$ mice) revealed no change in expression of $\beta$ CaMKII and $\alpha$ CaMKII in the Camk $2 b^{A 303 R}$ mouse (Fig. $4 c, d$ ). Since binding of calcium/calmodulin is a prerequisite for autophosphorylation of CaMKII at Thr286/287 (for review, see Hudmon and Schulman, 2002b; Lisman et al., 2002; Colbran, 2004), we used the phospho-Thr286/287 antibody to confirm that this mutation renders the $\beta$ CaMKII-A303R protein insensitive to calcium/calmodulin activation. Indeed, Western blot analysis showed that $\beta$ CaMKII Thr ${ }^{287}$ phosphorylation was entirely absent in the Camk $2 b^{A 303 R}$ mouse (Fig. $4 d$ ), confirming in vitro studies that the A303R mutation blocks activation of $\beta$ CaMKII. We also observed a significant reduction of $\alpha$ CaMKII $\mathrm{Thr}^{286}$ autophosphorylation (wild-type mice, $100 \pm 7.2, n=7$; Camk2 $b^{A 303 R}$ mice, 69.9 $\pm 8.3, n=5$; Mann-Whitney $U$ test, $U=$ $4.0, p=0.03$ ) (Fig. $4 d$ ), which is not unexpected given that most of the $\alpha$ CaMKII subunits are associated with $\beta$ CaMKII subunits, and that it takes two adjacent activated CaMKII subunits to get inter-subunit autophosphorylation at Thr286/Thr287 (Hudmon and Schulman, 2002b; Lisman et al., 2002; Colbran, 2004). Thionin staining did not reveal any gross morphological changes in 
the brain (Fig. 4e), indicating that development of the brain is normal despite the presence of an inactive form of $\beta$ CaMKII.

$\alpha$ CaMKII shows normal subcellular distribution in $\mathbf{C a m k} 2 \boldsymbol{b}^{A 303 R}$ neurons To directly examine the influence of $\beta$ CaMKII on the localization of $\alpha$ CaMKII, we tested whether synaptic targeting of $\alpha$ CaMKII was altered in neurons of the Camk2 $b^{A 303 R}$ mice. Remarkably, and in strong contrast to the findings in Camk $2 b^{-1-}$ neurons, Camk $2 b^{A 303 R}$ neurons showed normal $\alpha$ CaMKII synaptic localization $\left(\alpha\right.$ CaMKII $_{\text {synapse }} / \alpha$ CaMKII $_{\text {shaft }}$ : wild-type neurons, $4.2 \pm 0.7, n=6$; Camk2 $b^{A 303 R}$ neurons, $3.9 \pm 0.6, n=7$; Mann-Whitney $U$ test, $U=14.00, p=$ 0.37) (Fig. 5). These results demonstrate that $\beta$ CaMKII protein but not its calcium/ calmodulin-dependent activation is required for targeting $\alpha \mathrm{CaMKII}$ to synapses.

\section{The Camk $2 b^{A 303 R}$ mice show normal} LTP Camk $2 b^{A 303 R}$ mice retain normal synaptic localization of $\alpha$ CaMKII, despite a complete abrogation of calcium/calmodulindependent kinase activity of $\beta$ CaMKII. This provides a unique opportunity to dissect the mechanism by which $\beta$ CaMKII influences synaptic plasticity and learning.

As observed in Camk2 $b^{-1-}$ mice (Fig. $3 a$ ), extracellular recordings in acute hippocampal slices of $C a m k 2 b^{A 303 R}$ mice showed a slight, but not significant, reduction in basal synaptic transmission (basal synaptic transmission: repeated-measures ANOVA fiber volley, $F_{(1,46)}=0.44$, $p=0.51$; fEPSP slope, $F_{(1,46)}=0.93 ; p=0.34 ; n=25$ and 23, respectively, for wild-type and Camk2 $b^{A 303 R}$ mice) (Fig. 6a). However, in contrast to the Camk2b $b^{-1-}$ mice, which have a severe deficit in $100 \mathrm{~Hz} \mathrm{LTP}, C_{a m} 2 b^{A 303 R}$ mice showed normal $100 \mathrm{~Hz}$ LTP (wild-type mice, $149.7 \pm 9.2, n=15$; Camk $2 b^{A 303 R}$ mice, $133.4 \pm 7.5, n=10$; two-tailed $t$ test, $t_{(23)}=1.40, p=0.17$ ) (Fig. 6b). These results indicate that the kinase activity of $\beta$ CaMKII is dispensable for hippocampal synaptic plasticity and suggest that $\beta$ CaMKII functions principally to regulate the targeting of $\alpha \mathrm{CaMKII}$ into dendritic spines.

\section{Contextual fear learning of the $\operatorname{Camk} 2 \boldsymbol{b}^{A 303 R}$ mice}

Given that Camk $2 b^{-1-}$ mice showed a significant deficit in contextual fear conditioning (Fig. 3e), the results from Camk $2 b^{A 303 R}$ mice provide an opportunity to determine the mechanism of $\beta$ CaMKII function in hippocampus-dependent learning. Camk $2 b^{A 303 R}$ mice showed indistinguishable freezing behavior from their wild-type littermates (wild-type mice, $62.6 \pm 4.1, n=13$; $\operatorname{Camk}_{2} b^{A 303 R}$ mice, $53.5 \pm 5.0 n=11$; two-tailed $t$ test, $t_{(22)}=1.42, p=0.17$ ) (Fig. $6 c$ ) without any change in baseline freezing behavior (wild-type mice, $3.5 \pm 0.8, n=13$; Camk $2 b^{A 303 R}$ mice, $4.6 \pm 1.6, n=11$; MannWhitney $U$ test, $U=65.00, p=0.73$ ) (Fig. $6 c$ ). Hence, together with the results obtained using Camk $2 b^{-1-}$ mice, these results suggest that $\beta$ CaMKII-dependent localization of $\alpha \mathrm{CaMKII}$ is required for normal hippocampus-dependent learning. b

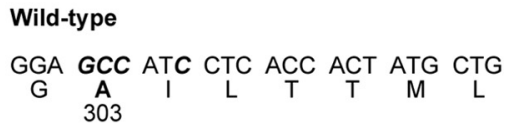

A303R mutation

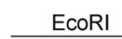

GGA AGA ATT CTC ACC ACT ATG CTG

$\begin{array}{llllllllllllll}G & R & \text { I } & \text { L } & T & T & M & \text { L }\end{array}$

e

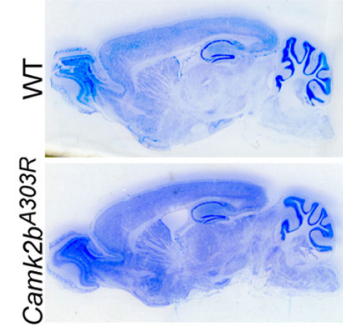

Figure 4. Generation of Camk $2 b^{A 303 R}$ mice. $a$, Schematic diagram for the generation of the Camk $2 b^{A 303 R}$ mutants. Genome, Wild-type ( Ala303Arg c Immunocytochemistry staining using an antibody specific for $\beta$ aKII shows no difference in $\beta$ CaMKII staining in reduction of Thr286 phosphorylation, whereas the Thr287 phosphorylation is completely absent in the hippocampus of the Camk2 $b^{A 303 R}$ mice. $e$, Thionin staining showed no gross morphological difference in the brains of Camk $2 b^{A 303 R}$ mice.

\section{Discussion}

Through a genetic dissection in mice, we have identified that $\beta$ CaMKII functions at the Schaffer collateral-CA1 synapse principally to target $\alpha$ CaMKII into dendritic spines. We found that the complete loss of $\beta$ CaMKII protein leads to mislocalization of $\alpha$ CaMKII in cultured neurons. Furthermore, we found that loss of $\beta$ CaMKII severely impairs hippocampal learning and synaptic plasticity. In contrast, the $\beta$ CaMKII-A303R mutant provided an elegant dissection of $\beta$ CaMKII function, since it fully retains F-actin binding despite a loss of calcium/calmodulin-dependent kinase activity. Indeed, $\operatorname{Camk} 2 b^{A 303 R}$ mice showed proper $\alpha$ CaMKII localization, robust LTP, and normal learning. Since we found that the subcellular localization of $\alpha$ CaMKII is changed only in the absence of $\beta$ CaMKII, and that the LTP deficit in the absence of $\beta$ CaMKII can be overcome by using a strong LTP-inducing protocol, our data support a role of $\beta$ CaMKII, in which it regulates the targeting of $\alpha$ CaMKII into spines. Given the extensive literature showing that $\beta$ CaMKII has F-actin binding and bundling properties, which are fully conserved in the $\beta$ CaMKII-A303R mutant, we have concluded that the synaptic plasticity and learning deficits observed in the Camk $2 b^{-1-}$ mutants result from the impaired $\beta$ CaMKII-dependent targeting of $\alpha$ CaMKII into spines.

\section{$\beta C a M K I I$ is not required for neuronal development}

Considering the developmentally earlier expression of $\beta$ CaMKII compared with $\alpha$ CaMKII (Sahyoun et al., 1985; Bayer et al., 

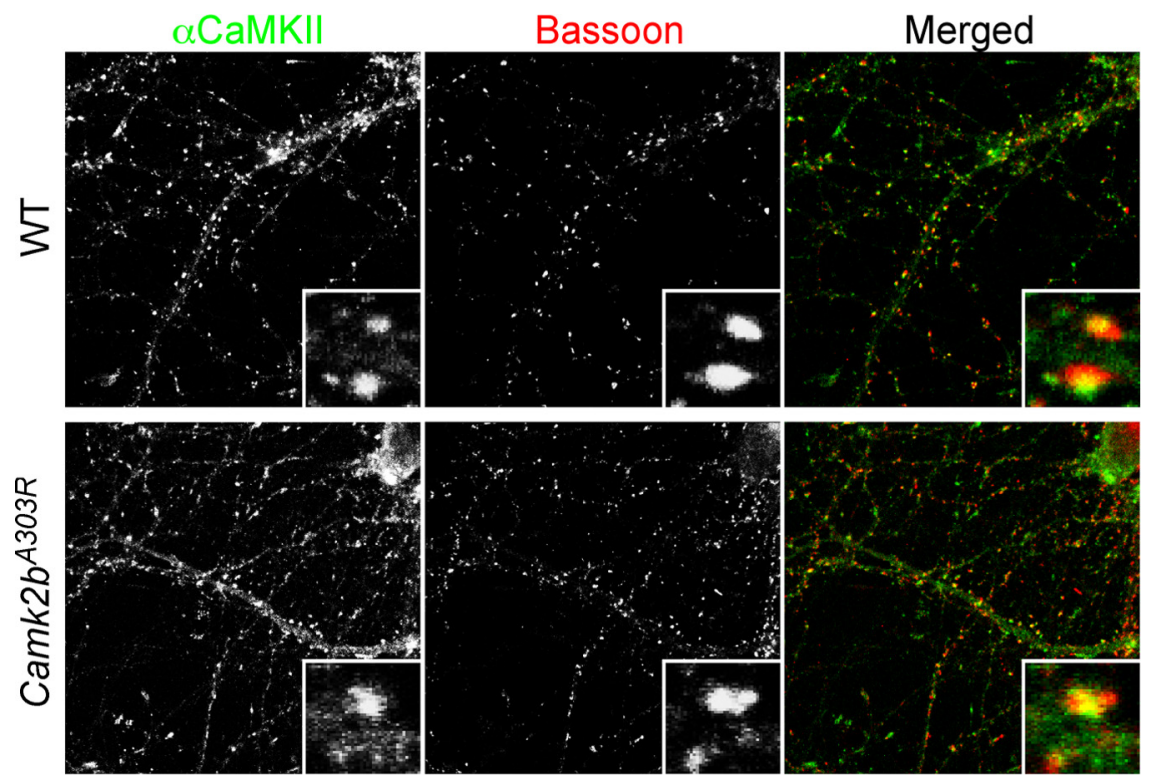

Figure 5. Normal distribution of $\alpha$ CaMKII in cultured neurons of Camk2 $b^{A 303 R}$ mice. Representative images of cultured wildtype and Camk2 ${ }^{A 303 R}$ hippocampal neurons labeled with $\alpha$ CaMKII (green) and bassoon (red), and their colocalization (merged) showing normal distribution of $\alpha$ CaMKII in Camk2 $b^{A 303 R}$ hippocampal neurons. Inserts show an enlargement of a dendritic segment with two spines.
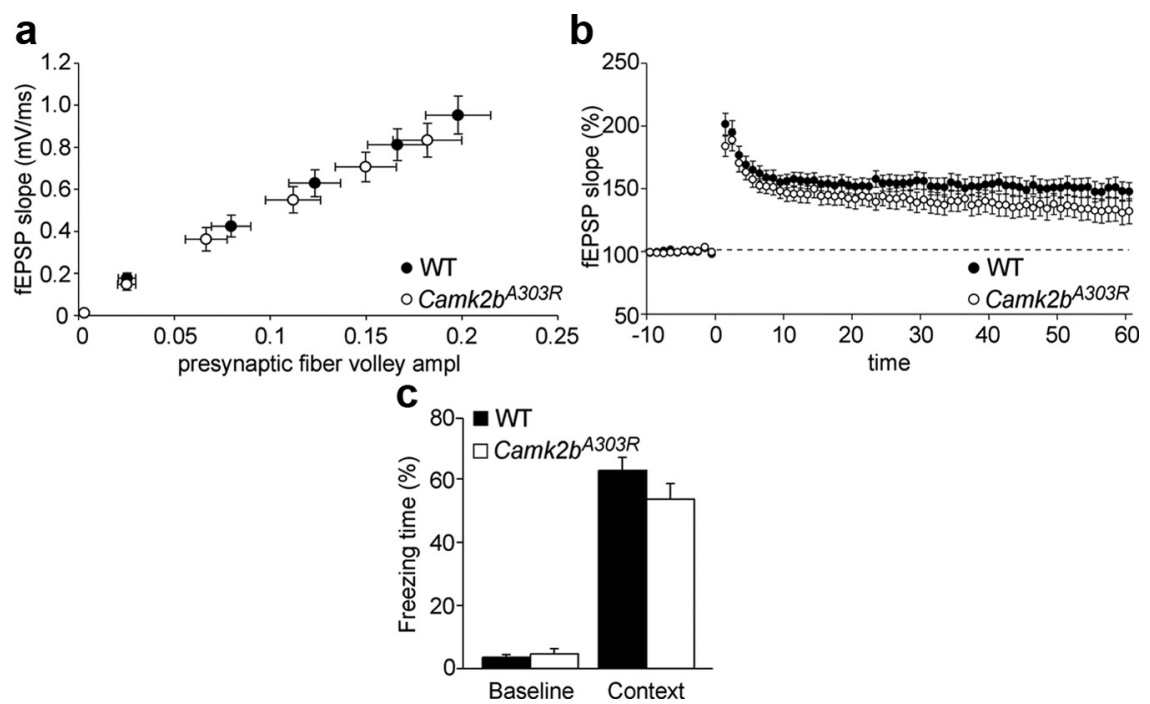

Figure 6. Normal hippocampal synaptic plasticity and learning of Camk $2 b^{A 303 R}$ mice. $a$, Camk $2 b^{A 303 R}$ mice show normal synaptic transmission (wild-type, $n=68$; $\left(a m k 2 b^{A 303 R}, n=58\right)$. b. Camk2 $b^{A 303 R}$ mice show normal $100 \mathrm{~Hz}$ LTP (wild-type, $n=28$; Camk2 $\left.b^{A 303 R}, n=19\right)$. $c$, Camk2 ${ }^{A 303 R}$ mice show normal contextual fear conditioning. Percentage of time spent freezing during training before the footshock (Pre) and $24 \mathrm{~h}$ after conditioning (Post), showing normal learning in Camk $2 b^{A 303 R}$ mice (wild-type, $n=14 ;$ ( Camk $\left.2 b^{A 303 R}, n=6\right)$. Error bars represent SEM.

1999) and the observation that knockdown or overexpression of $\beta C a M K I I$ affects synapse number and neurite outgrowth in cultured hippocampal neurons or neuroblastoma cells (Nomura et al., 1997; Fink et al., 2003), a large effect on hippocampal development could be expected. However, we found that neither the absence nor the inactivation of $\beta$ CaMKII affects brain development at the light microscopic level. Hence, we conclude that $\beta \mathrm{CaMKII}$ is not required for normal brain development and for normal spine number of CA1 pyramidal neurons. This is similar to previous findings in the cerebellum (van Woerden et al., 2009). However, although our data show that $\beta$ CaMKII is dispensable for normal brain development, we cannot rule out that germ line mutations in $\beta$ CaMKII result in homeostatic compensatory mechanisms to prevent the changes seen in neuronal cultures shortly after the expression of $\beta$ CaMKII was changed.

\section{$\beta$ CaMKII-dependent hippocampal synaptic plasticity}

We showed that $\beta$ CaMKII is required for normal hippocampal NMDA receptordependent plasticity as well as learning. Notably, despite the fact that $\beta$ CaMKII has a 10 -fold higher affinity for calcium/calmodulin compared with $\alpha$ CaMKII, the impairment in NMDA receptor-dependent LTP in Camk $2 b^{-1-}$ mice cannot be explained by a reduction in calcium/calmodulin sensitivity, since LTP deficits were not observed in the Camk $2 b^{A 303 R}$ mice, in which $\beta$ CaMKII has a negligible affinity for calcium/calmodulin. Rather, the deficit in NMDAdependent LTP is most likely caused by the reduced synaptic localization of $\alpha \mathrm{CaMKII}$, as our data show that the subcellular localization of $\alpha$ CaMKII is strongly influenced by $\beta$ CaMKII. Specifically, there is approximately a $50 \%$ reduction in the $\alpha$ CaMKII spine/shaft ratio in Camk $2 b^{-1-}$ neurons, whereas the localization of $\alpha$ CaMKII in Camk $2 b^{A 303 R}$ neurons is indistinguishable from wild-type mice. Hence, $\beta$ CaMKII functions independently of its calciumdependent kinase activity to regulate the synaptic localization of $\alpha$ CaMKII.

We realize that our study has some limitations. First, to be able to quantify the subcellular localization of $\alpha$ CaMKII we had to revert to (ex vivo) dissociated neuronal cultures obtained from the mutant mice, which is the commonly used technique to study CaMKII localization. Whether the observed changes also hold true in vivo remains to be shown. In addition, our localization studies cannot distinguish between presynaptic and postsynaptic $\alpha$ CaMKII. However, given the relative abundance of postsynaptic $\alpha \mathrm{CaMKII}$, and the important role of postsynaptic $\alpha$ CaMKII in LTP, we believe that our findings strongly suggest that $\beta$ CaMKII is particularly important for targeting postsynaptic CaMKII. Last, although the observed reduction of synaptic $\alpha$ CaMKII is likely underlying the NMDA-receptor-dependent LTP deficit, we cannot rule out that the loss of $\beta$ CaMKII also affects other synaptic processes associated with the $\beta$ CaMKII-dependent actin binding and bundling properties. But regardless of the precise nature of the deficits, it is interesting to note that the plasticity deficits can be overcome by a very strong LTP-inducing protocol consisting of four trains of $200 \mathrm{~Hz}$ (Grover and Teyler, 1990). Possibly, this protocol is able to activate the pool of $\alpha$ CaMKII, which is present in the shaft. In this respect, it is interesting to note that a recent study showed that voltage-gated calcium channels (VGCCs) are able to activate a different pool of $\alpha \mathrm{CaMKII}$ than NMDA receptors, with a stronger activation of $\alpha$ CaMKII in 
dendritic shafts compared with $\alpha$ CaMKII in spines (Lee et al., 2009). However, whether activation of VGCCs is responsible for the LTP rescue remains to be investigated.

Together, our data show that $\beta$ CaMKII is essential for hippocampus-dependent learning and for normal plasticity at the Schaffer collateral-CA1 synapse. Despite the fact that $\beta$ CaMKII has a higher affinity for calcium/calmodulin compared with $\alpha$ CaMKII, we found that the calcium/calmodulin-dependent activation of $\beta$ CaMKII was fully dispensable for hippocampal LTP and learning. Rather, our data show that $\beta$ CaMKII in hippocampal pyramidal neurons plays a structural role, which serves to target $\alpha$ CaMKII to synapses.

\section{References}

Allison DW, Chervin AS, Gelfand VI, Craig AM (2000) Postsynaptic scaffolds of excitatory and inhibitory synapses in hippocampal neurons: maintenance of core components independent of actin filaments and microtubules. J Neurosci 20:4545-4554.

Bayer KU, Löhler J, Schulman H, Harbers K (1999) Developmental expression of the CaM kinase II isoforms: ubiquitous gamma- and delta-CaM kinase II are the early isoforms and most abundant in the developing nervous system. Brain Res Mol Brain Res 70:147-154.

Brocke L, Chiang LW, Wagner PD, Schulman H (1999) Functional implications of the subunit composition of neuronal CaM kinase II. J Biol Chem 274:22713-22722.

Cho MH, Cao X, Wang D, Tsien JZ (2007) Dentate gyrus-specific manipulation of beta-Ca2+/calmodulin-dependent kinase II disrupts memory consolidation. Proc Natl Acad Sci U S A 104:16317-16322.

Colbran RJ (2004) Targeting of calcium/calmodulin-dependent protein kinase II. Biochem J 378:1-16.

De Koninck P, Schulman H (1998) Sensitivity of CaM kinase II to the frequency of Ca2+ oscillations. Science 279:227-230.

Elgersma Y, Fedorov NB, Ikonen S, Choi ES, Elgersma M, Carvalho OM, Giese KP, Silva AJ (2002) Inhibitory autophosphorylation of CaMKII controls PSD association, plasticity, and learning. Neuron 36:493-505.

Elgersma Y, Sweatt JD, Giese KP (2004) Mouse genetic approaches to investigating calcium/calmodulin-dependent protein kinase II function in plasticity and cognition. J Neurosci 24:8410-8415.

Fink CC, Bayer KU, Myers JW, Ferrell JE Jr, Schulman H, Meyer T (2003) Selective regulation of neurite extension and synapse formation by the beta but not the alpha isoform of CaMKII. Neuron 39:283-297.

Frankland PW, O'Brien C, Ohno M, Kirkwood A, Silva AJ (2001) AlphaCaMKII-dependent plasticity in the cortex is required for permanent memory. Nature 411:309-313.

Giese KP, Fedorov NB, Filipkowski RK, Silva AJ (1998) Autophosphorylation at Thr286 of the alpha calcium-calmodulin kinase II in LTP and learning. Science 279:870-873.

Goslin K, Banker G, eds (1991) Culturing nerve cells. Cambridge, MA: MIT.

Grover LM, Teyler TJ (1990) Two components of long-term potentiation induced by different patterns of afferent activation. Nature 347:477-479.

Hansel C, de Jeu M, Belmeguenai A, Houtman SH, Buitendijk GH, Andreev D, De Zeeuw CI, Elgersma Y (2006) $\alpha$ CaMKII is essential for cerebellar LTD and motor learning. Neuron 51:835-843.

Hudmon A, Schulman H (2002a) Structure-function of the multifunctional $\mathrm{Ca} 2+/$ calmodulin-dependent protein kinase II. Biochem J 364:593-611.

Hudmon A, Schulman H (2002b) Neuronal CA2+/calmodulin-dependent protein kinase II: the role of structure and autoregulation in cellular function. Annu Rev Biochem 71:473-510.

Jaworski J, Kapitein LC, Gouveia SM, Dortland BR, Wulf PS, Grigoriev I, Camera P, Spangler SA, Di Stefano P, Demmers J, Krugers H, Defilippi P, Akhmanova A, Hoogenraad CC (2009) Dynamic microtubules regulate dendritic spine morphology and synaptic plasticity. Neuron 61:85-100.
Lee SJ, Escobedo-Lozoya Y, Szatmari EM, Yasuda R (2009) Activation of CaMKII in single dendritic spines during long-term potentiation. Nature 458:299-304.

Lin YC, Redmond L (2008) CaMKIIbeta binding to stable F-actin in vivo regulates F-actin filament stability. Proc Natl Acad Sci U S A 105:15791-15796.

Lisman J, Schulman H, Cline H (2002) The molecular basis of CaMKII function in synaptic and behavioural memory. Nat Rev Neurosci 3:175-190.

Mayford M, Bach ME, Huang YY, Wang L, Hawkins RD, Kandel ER (1996) Control of memory formation through regulated expression of a CaMKII transgene. Science 274:1678-1683.

Miller S, Yasuda M, Coats JK, Jones Y, Martone ME, Mayford M (2002) Disruption of dendritic translation of CaMKIIalpha impairs stabilization of synaptic plasticity and memory consolidation. Neuron 36:507-519.

Nomura T, Kumatoriya K, Yoshimura Y, Yamauchi T (1997) Overexpression of alpha and beta isoforms of $\mathrm{Ca} 2+/$ calmodulin-dependent protein kinase II in neuroblastoma cells- $-\mathrm{H}-7$ promotes neurite outgrowth. Brain Res 766:129-141.

Okamoto K, Nagai T, Miyawaki A, Hayashi Y (2004) Rapid and persistent modulation of actin dynamics regulates postsynaptic reorganization underlying bidirectional plasticity. Nat Neurosci 7:1104-1112.

Okamoto K, Narayanan R, Lee SH, Murata K, Hayashi Y (2007) The role of CaMKII as an F-actin-bundling protein crucial for maintenance of dendritic spine structure. Proc Natl Acad Sci U S A 104:6418-6423.

O'Leary H, Lasda E, Bayer KU (2006) CaMKIIbeta association with the actin cytoskeleton is regulated by alternative splicing. Mol Biol Cell 17:4656-4665.

Sahyoun N, LeVine H 3rd, Burgess SK, Blanchard S, Chang KJ, Cuatrecasas P (1985) Early postnatal development of calmodulin-dependent protein kinase II in rat brain. Biochem Biophys Res Commun 132:878-884.

Sanabria H, Swulius MT, Kolodziej SJ, Liu J, Waxham MN (2009) $\beta$ CaMKII regulates actin assembly and structure. J Biol Chem 284:9770-9780.

Shen K, Meyer T (1999) Dynamic control of CaMKII translocation and localization in hippocampal neurons by NMDA receptor stimulation. Science 284:162-166.

Shen K, Teruel MN, Subramanian K, Meyer T (1998) CaMKIIbeta functions as an F-actin targeting module that localizes CaMKIIalpha/beta heterooligomers to dendritic spines. Neuron 21:593-606.

Silva AJ, Stevens CF, Tonegawa S, Wang Y (1992) Deficient hippocampal long-term potentiation in alpha-calcium-calmodulin kinase II mutant mice. Science 257:201-206.

Tao-Cheng JH, Vinade L, Pozzo-Miller LD, Reese TS, Dosemeci A (2002) Calcium/calmodulin-dependent protein kinase II clusters in adult rat hippocampal slices. Neuroscience 115:435-440.

Thiagarajan TC, Piedras-Renteria ES, Tsien RW (2002) alpha- and betaCaMKII. Inverse regulation by neuronal activity and opposing effects on synaptic strength. Neuron 36:1103-1114.

van Woerden GM, Harris KD, Hojjati MR, Gustin RM, Qiu S, de Avila Freire R, Jiang YH, Elgersma Y, Weeber EJ (2007) Rescue of neurological deficits in a mouse model for Angelman syndrome by reduction of alphaCaMKII inhibitory phosphorylation. Nat Neurosci 10:280-282.

van Woerden GM, Hoebeek FE, Gao Z, Nagaraja RY, Hoogenraad CC, Kushner SA, Hansel C, De Zeeuw CI, Elgersma Y (2009) betaCaMKII controls the direction of plasticity at parallel fiber-Purkinje cell synapses. Nat Neurosci 12:823-825.

Wang H, Feng R, Phillip Wang L, Li F, Cao X, Tsien JZ (2008) CaMKII activation state underlies synaptic labile phase of LTP and short-term memory formation. Curr Biol 18:1546-1554.

Yamagata Y, Kobayashi S, Umeda T, Inoue A, Sakagami H, Fukaya M, Watanabe M, Hatanaka N, Totsuka M, Yagi T, Obata K, Imoto K, Yanagawa Y, Manabe T, Okabe S (2009) Kinase-dead knock-in mouse reveals an essential role of kinase activity of $\mathrm{Ca}^{2+} /$ calmodulin-dependent protein kinase II $\alpha$ in dendritic spine enlargement, long-term potentiation, and learning. J Neurosci 29:7607-7618. 